\title{
Autocuidado para el control de la hipertensión arterial en adultos mayores ambulatorios: una aproximación a la taxonomía NANDA-NOC-NIC*
}

\section{Self-Care for hypertension in elderly outpatients, an approach to the NANDA-NOC-NIC taxonomy}

Rey Arturo Salcedo-Álvarez,

Blanca Consuelo González-Caamaño..•

Araceli Jiménez Mendoza...

Vanessa Nava Ríos, Dheeni Azyade Cortés Olguín, Martha Belem Eugenio Retana....

\section{Resumen}

El uso de un lenguaje común es una característica que distingue a los profesionales. Así, la conformación y el uso de un lenguaje técnico coadyuva al desarrollo gremial, en este sentido hacer uso de las teorías del cuidado y de clasificaciones validadas por asociaciones internacionales de profesionales sintetizan y facilitan la comprensión y el avance del conocimiento.

Cuando los profesionales de enfermería aplican teorías y clasificaciones para discutir sobre la gestión del cuidado, el nivel de comunicación mejora y los pacientes se ven beneficiados con la continuidad de su tratamiento.

El propósito de éste trabajo, es mostrar de manera explícita como se puede vincular la teoría del autocuidado de Orem, con los diagnósticos de enfermería propuestos por la Asociación Norteamericana de Diagnósticos de Enfermería; la Clasificación de Resultados de Enfermería; y la Clasificación de Intervenciones de Enfermería.

Con base en lo anterior, se proponen cuatro esquemas que pueden ser aplicables al cuidado de adultos mayores ambulatorios con hipertensión arterial.

* Proyecto 049 ENEO-UNAM: Efecto de una intervención educativa en la calidad de vida de los adultos mayores con hipertensión arterial GRUPO DE INVESTIGACIÓN EN GESTIÓN DEL CUIDADO - Profesor de Carrera Asociado "C" ENEO-UNAM, ..Profesor de Asignatura "A" ENEO-UNAM. ...Profesor de Carrera Titular "B" ENEO-UNAM. ....Pasantes de la Licenciatura en Enfermería y Obstetricia en Servicio Social ENEO-UNAM. Correspondencia: Rey Arturo Salcedo Álvarez. Camino viejo a Xochimilco S/N y Viaducto. Tlalpan Col. San Lorenzo Huipulco, C. P. 14370 México D. F. Correo electrónico: rasalced@hotmail.com

Fecha de Recibido: 19 MAYO 2012

FECHA ENVIADO: 26 MAYO 2012

FECHA ACEPTADO: 29 JUNIO 2012

Palabras Clave:

Taxonomía, NIC, NOC, hipertensión arterial, adultos mayores y autocuidado. 


\section{Enfermería Universitaria}

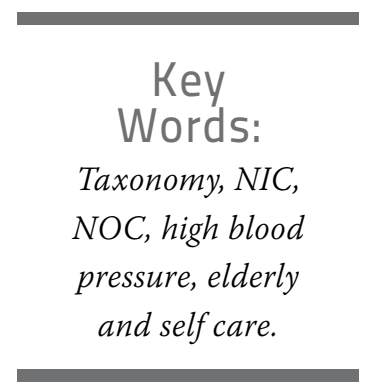

\section{AbSTRAC}

A characteristic among professionals is the use of common language. Therefore the conformation of a technical language usage contributes to professional development, in this way, the use of care theories and classifications validated by international professional associations summarize and facilitate the comprehension and advance of knowledge.

When nursing professionals apply theories and classifications to discuss about care management, the level of communication improves and patients get favored with the continuity of their treatment.

The purpose of this paper work is to show in an explicit manner how Orem's theory of self care can be linked with nursing diagnosis purposed by the North American Nursing Diagnosis Association, the Nursing Outcomes Classification and the Nursing Interventions Classification.

Based on previous said, five diagrams are suggested to be applied in the care of elderly outpatients with high blood pressure.

\section{INTRODUCCIÓN}

Parte importante en la innovación de la práctica profesional es la gestión del cuidado, misma que se define como: "la aplicación de un juicio profesional en la planificación, organización, motivación y control de la provisión de cuidados, oportunos, seguros, integrales, que aseguren la continuidad de la atención y se sustenten en las políticas y lineamientos estratégicos de las instituciones prestadoras de servicios de salud, propiciando el uso adecuado de los recursos para el mejor cuidado de las personas." Asimismo, implica también la sistematización y esquematización de experiencias en la atención de los pacientes. El propósito de éste trabajo es mostrar cómo a partir de la taxonomía de los diagnósticos de enfermería de la NANDA, ${ }^{2}$ la Clasificación de Resultados de Enfermería (NOC) ${ }^{3}$ y la Clasificación de Intervenciones de Enfermería (NIC) $)^{4}$ se puede construir un esquema que permita visualizar la planeación, la ejecución y la evaluación de los avances en la atención de los adultos mayores (AM) con hipertensión arterial (HTA).

\section{Adultos mayores e hipertensión arterial}

La relevancia de estudiar AM e HTA es que este grupo poblacional es el de más rápido crecimiento $y$ el que mayores necesidades de salud presenta, en nuestro país para 2001 acumulaban el 5\% de la población total y se calculaba que en 2030 alcanzarían el $12.6 \% .^{5} \mathrm{Sin}$ embargo, datos del último censo ${ }^{6}$ indican que en 2010 la población total fue de 112, 322,757 de los cuales 9, 890,455 eran AM por lo que la proporción fue de $8.8 \%$, este 
incremento de $3.8 \%$ en tan sólo nueve años nos hace pensar que muy probablemente la estimación que se había hecho para la tercer década de este tercer milenio suceda antes. El Consejo Nacional de Población estima que a nivel mundial la población de AM ascienda a 16.6 por ciento de la población en el año 2030 y para el 2050 a 21.4 por ciento.?

La hipertensión arterial es un padecimiento crónico degenerativo, cardiovascular, caracterizado por el aumento de las cifras consideradas como normales en cuanto a los valores de la presión sistólica y diastólica que se miden en el ser humano. Se presenta principalmente en la población con edad avanzada y afecta órganos vitales como el riñón, sistema nervioso central, ojos corazón y sistema vascular. $^{8}$

En los AM mexicanos esta enfermedad está presente en el 34.7\% de los hombres y en el $49.0 \%$ de las mujeres, ${ }^{9}$ el estudio de base poblacional más reciente es la Encuesta Nacional de Salud y Nutrición $2006^{10}$ en ella se muestra que la prevalencia de HTA por autoreporte en AM fue de $28.5 \%$ para hombres y $41.3 \%$ para mujeres, esta encuesta reconoce también que la HTA es uno de los factores de riesgo más importantes de enfermedad cardiovascular y una de las principales causas de mortalidad.

La participación del personal de enfermería en el tratamiento de la hipertensión arterial es determinante para propiciar los cambios en el estilo de vida del individuo y su familia que se requieren para minimizar los factores de riesgo cardiovascular; dentro de las medidas protectoras se encuentran:

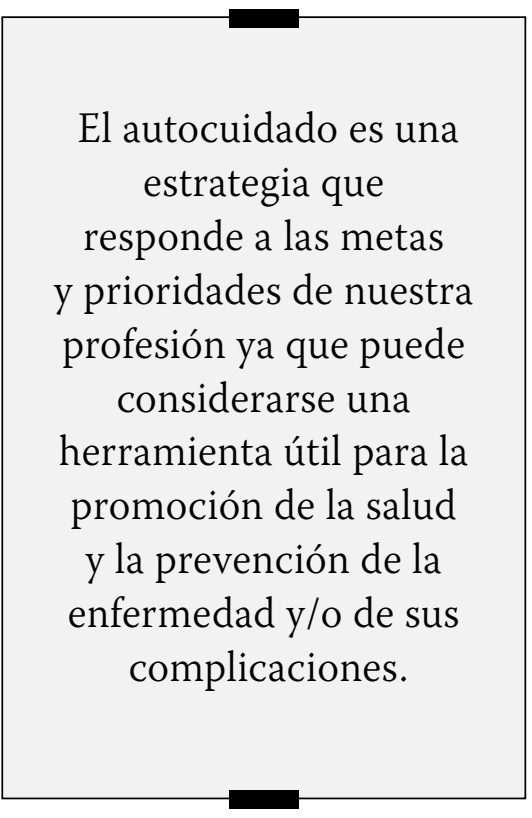

la realización de ejercicio físico, adaptación a nuevos regímenes nutricionales, mantenimiento de peso ideal, disminución del consumo de licor y de cigarrillo, adherencia al tratamiento farmacológico y el manejo de respuestas psicológicas.

Por ser éste un tema concerniente a la disciplina, el autocuidado se ha convertido en un tema de investigación cada vez de mayor actualidad. Desde la perspectiva de enfermería el autocuidado es una estrategia que responde a las metas y prioridades de nuestra profesión ya que puede considerarse una herramienta útil para la promoción de la salud y la prevención de la enfermedad y/o de sus complicaciones, auxiliándose preferentemente de teorías de enfermería, como es el caso de la teoría del autocuidado de la enfermera Dorothea Orem. ${ }^{11}$

La Teoría General de Autocuidado de Dorothea Orem comprende tres teorías: la teoría del autocuidado, la teoría del déficit del autocuidado y la teoría del sistema de enfermería.

\section{Teoría del autocuidado}

Para el desarrollo de este apartado tomaremos como referencia básica el libro original del Modelo de Orem $^{12}$. Para Orem, enfermería debe identificar las capacidades potenciales de autocuidado del individuo para que ellos puedan satisfacer sus necesidades de autocuidado con el fin de mantener la vida y la salud, recuperarse de los daños y de la enfermedad y manejarse con sus efectos. El foco de enfermería es identificar el déficit entre la capacidad potencial de autocuidado y las demandas de autocuidado de los pacientes. La meta es eliminar este, de tal forma que se cubran los requerimientos/necesidades universales del desarrollo y se limiten las desviaciones en la salud.

Los cuidados de enfermería se orientan en sistemas de enfermería donde se distinguen tres niveles de participación: a) totalmente compensatorio (la enfermera realiza todo el autocuidado del paciente); b) sistema parcialmente compensatorio (enfermera y paciente realizan el autocuidado) y c) el de apoyo educativo (la enfermera ayuda a superar cualquier limitación en el autocuidado).

La enfermera proporciona asistencia especializada a personas con incapacidades tales que requieren más de una ayuda común para cubrir las necesidades diarias de autocuidado y para participar inteligentemente en los cuidados que recibe del Personal de salud.

Para Orem "El autocuidado es la práctica de actividades que los individuos realizan en su propio beneficio para el mantenimiento de la vida, la salud y el bienestar". 
Específicamente puede considerarse que un individuo cuida de sí mismo si realiza efectivamente las siguientes actividades:

1. Apoyo de los procesos vitales y el funcionamiento normal.

2. Mantenimiento del crecimiento, maduración y desarrollo normales.

3. Prevención o control de los procesos de enfermedad o lesiones.

4 Prevención de incapacidad o su compensación.

5. Promoción del bienestar.

\section{Requisitos de autocuidado}

Orem ha identificado y descrito ocho actividades que son esenciales o imprescindibles para ejercer el autocuidado, independientemente del estado de salud, edad, nivel de desarrollo o entorno ambiental de un individuo. Estas actividades, por ese carácter imprescindible, son llamadas requisitos de cuidado universal y comprenden:

Mantenimiento de un aporte suficiente de aire, mantenimiento de un aporte suficiente de agua, mantenimiento de un soporte suficiente de alimentos, provisión de cuidados asociados con los procesos de eliminación y los excrementos, mantenimiento del equilibrio entre la actividad y el reposo, mantenimiento del equilibrio entre la soledad y la interacción social, prevención de peligros para la vida, el funcionamiento humano y el bienestar humano y promoción del funcionamiento y desarrollo humano dentro de los grupos sociales de acuerdo con el potencial humano, las limitaciones humanas conocidas y deseo humano de ser normal (normalidad).

Estas actividades de autocuidado universal abarcan los elemen- tos físicos, psicológicos, sociales y espirituales esenciales de la vida. Cada uno de ellos importante para el funcionamiento humano.

\section{Requisitos de autocuidado del desarrollo}

Además de las actividades de autocuidado universal, esenciales para todas las personas en todas las etapas del desarrollo, Orem identificó un segundo tipo de actividades asociadas con el desarrollo humano, que se presentan en circunstancias especiales y en ciertas etapas específicas, como el ser un neonato. En tales etapas debe de presentarse una especial consideración a aspectos del cuidado necesarios para sostener la vida y dirigidos específicamente a la promoción del desarrollo.

Este segundo grupo implica la provisión de cuidados asociados con condiciones que pueden afectar adversamente el desarrollo humano. Este grupo se divide a su vez en dos subtipos; el primero se refiere a la provisión de cuidados para prevenir los efectos negativos de estas condiciones adversas, el segundo se refiere a la provisión de cuidados para mitigar o superar los efectos negativos existentes (o potenciales) de una condición o acontecimientos vitales específicos. Situaciones relevantes que pueden interferir en el desarrollo humano o en la capacidad de autocuidado y por tanto deben ser consideradas son:

1. Deprivación educacional

2. Problemas de adaptación social

3. Pérdida de familiares, amigos o colaboradores

4. Pérdida de posesiones o del trabajo
Las actividades de autocuidado universal abarcan los elementos físicos, psicológicos, sociales

y espirituales esenciales de la vida. Cada uno de ellos importante para el funcionamiento humano.

5. Cambio súbito de condiciones de vida

6. Cambio de posición ya sea social o económica

7. Mala salud, malas condiciones de vida o incapacidad

8. Enfermedad terminal o muerte esperada

9. Peligros ambientales

\section{Requisitos de autocuidado} en la desviación de la salud Cuando el individuo está enfermo, sufre alguna lesión, tiene incapacidades o está recibiendo atención médica tiene las siguientes demandas adicionales de cuidados de la salud:

Buscar y asegurar la ayuda médica apropiada en caso de exposición a condiciones ambientales o agentes físicos o biológicos específicos asociados con acontecimientos y estados patológicos humanos, o cuando hay indicios o condiciones genéticas, fisiológicas o psicológicas que determinan patología humana o están asociadas a esta. 
Ser consciente de los efectos y resultados de las condiciones y estados patológicos, incluyendo los efectos sobre el desarrollo, y atender a ellos.

Realizar eficazmente las medidas diagnósticas, terapéuticas y de rehabilitación previamente prescrita, dirigida a la prevención de tipos específicos de patología, a la propia patología, a la regulación del funcionamiento humano integrado, a la corrección de deformidades o anomalías, o a la compensación de incapacidades.

Ser consciente de los efectos negativos de las medidas terapéuticas realizadas o prescritas por el médico, incluyendo su influencia sobre el desarrollo y atender dichos efectos o regularlos.

Modificación del autoconcepto (y autoimagen) aceptándose uno mismo como ser con un estado particular de salud y que necesita formas específicas de cuidados de salud.

Aprender a vivir con los efectos de condiciones y estados patológicos y los efectos de las medidas diagnósticas y terapéuticas, en un estilo de vida que promueva el desarrollo personal continuado. ${ }^{12}$

\section{Factores condicionantes básicos para el autocuidado}

Las demandas de autocuidado terapéutico de las personas cuyo estado de salud está dentro de las normas de auto cuidados requeridos, se denominan factores básicos condicionantes, los cuales están constituidos por los requisitos (actividades) de autocuidado universal y los de autocuidado del desarrollo. Estos factores básicos de autocuidado tienen su fundamento en la naturaleza de los seres humanos como son: Edad, sexo, estado de desarrollo, estado de salud, orientación sociocultural, factores del sistema de cuidados de salud, factores del sistema familiar, patrón de vida, factores ambientales y disponibilidad y adecuación de los recursos.

Conforme a estos planteamientos centrales y dado que todavía no existen estudios suficientes en la población más anciana-mayores de 85 años, se ha propuesto que el tratamiento individualizado es el que debería realizarse en nuestra práctica clínica vital. ${ }^{13}$

\section{Diagnósticos, Intervenciones y Resultados de Enfermería}

Para la realización de este trabajo se utilizaron los "Diagnósticos de Enfermería” propuestos por la North American Nursing Diagnosis Association (NANDA); para los "Resultados de enfermería" aplicamos los propuestos por la Nursing Outcomes Clasification (NOC); y para las "Intervenciones de Enfermería" empleamos la propuesta de la Nursing Interventions Clasification (NIC). ${ }^{14}$

\section{Plan de Atención de acuerdo a los Diagnósticos de Enfermería} Como se mencionó la teoría General de Autocuidado de Dorothea Orem comprende tres teorías; la teoría del autocuidado de la cuál seleccionamos los requisitos universales, requisitos de desarrollo y

Todavía no existen estudios suficientes en la población mayor de 85 años, el tratamiento individualizado es el que debería realizarse en nuestra práctica clínica vital requisitos de desviación de la salud de mayor relevancia en el AM con HTA, la teoría del déficit del autocuidado que menciona al Agente y a la Agencia de autocuidado que en este caso será la enfermera y la teoría de sistemas de enfermería que pueden ser totalmente compensatorios, parcialmente compensatorios y de apoyo/ educación siendo este el que se utilizó para elaborar el plan de atención para el AM con HTA. ${ }^{15}$

Se diseñaron cuatro esquemas para el manejo del paciente ambulatorio con HTA, dichos instrumentos fueron estructurados con base a las cinco etapas del proceso enfermero; para la etapa de valoración se identificaron las necesidades educativas más frecuentes en este tipo de pacientes; en la de diagnóstico se eligieron las etiquetas que se vincularon con dichas necesidades y se elaboraron diagnósticos de enfermería con base al formato etiqueta diagnóstica (problema); $\mathrm{r} / \mathrm{c}$ factor relacionado (etiología); y $\mathrm{m} / \mathrm{p}$ manifestaciones (signos $\mathrm{y}$ síntomas); posteriormente para la etapa de planeación se muestran los "Resultados de enfermería" con sus respectivos indicadores y escalas de medición que van en relación y congruencia con la etiqueta diagnóstica y/o factor relacionado.

Para la etapa de ejecución se utilizaron las "Intervenciones de enfermería" donde se ejemplifican las intervenciones y actividades más adecuadas para contribuir a la implementación del tratamiento de enfermería que para esta investigación consistirán en las enseñanzas de autocuidado para el control de la HTA.

Finalmente, para la evaluación se utilizarán los indicadores específicos de cada diagnóstico de 
acuerdo a la clasificación NOC, para permitir evaluar la efectividad de las intervenciones de enfermería con base al resultado esperado propuesto en la etapa de planeación y por consiguiente establecer si existió mejoría o no en el estado de salud del paciente.

Por último se diseñaron los esquemas para vincular las taxonomías NANDA, NOC y NIC con la información anterior (Cuadros 1, 2, 3 y 4 Anexo al final del artículo).

\section{Conclusiones}

Los AM son el grupo poblacional de más rápido crecimiento y también el que presenta mayores problemas y necesidades de salud, se estima que hasta una tercera parte de ellos están afectados por padecimientos crónicos como lo es la hipertensión arterial, entidad que si bien por definición no es curable, si es controlable con cuidados, enseñanza del cuidado y autocuidado en donde el personal de enfermería ostenta un papel preponderante.

La sistematización de las acciones para el cuidado basadas en criterios validados por asociaciones gremiales coadyuva a la generación de un lenguaje que más allá de profesionalizar la práctica, incrementa la calidad de la atención al permitir el seguimiento del cuidado, situación que beneficia a los pacientes.

Adicionalmente, utilizar teorías disciplinares como es el caso de la propuesta por Dorothea Orem, favorece la mejor comprensión de aspectos filosóficos, teóricos y conceptuales inherentes al cuerpo de conocimientos enfermeros con un sentido humanista; visualizar esquemáticamente la concepción del cuidado científico fortalece al gremio enfermero.
Si bien la finalidad de este trabajo fue mostrar una aplicación práctica del uso que se le puede dar a la taxonomía de los diagnósticos de enfermería NANDA, la Clasificación de Resultado de Enfermería (NOC) y la Clasificación de Intervenciones de Enfermería (NIC) en la gestión del cuidado de adultos mayores con hipertensión arterial; la propuesta es que utilizando este tipo de esquemas, investigadoras de otras áreas del cuidado desarrollen nuevos diseños para otros problemas de salud a fin de sistematizar, esquematizar y compartir éstas experiencias en la atención de los pacientes.

\section{REFERENCIAS BIBLIOGRÁFICAS}

1 Norma General Administrativa No 19, Gestión del Cuidado de Enfermería para la Atención Cerrada. mht. Resolución 1127.07.2007 doc. Santiago, 14, 1.12.2007. Disponible en: http://bit.ly/Vh8fC9

2 North American Nursing Diagnosis Association. NANDA-I Diagnósticos enfermeros: Definiciones y Clasificación 2009-2011. Barcelona: Elsevier, 2010.

3 Moorhead S, Johnson M, Maas M, Swanson E. Clasificación de Resultados de Enfermería (NOC) $4^{\text {a }}$ edición. Barcelona: Elsevier, 2009.

4 Bulecheck G, Butcher H, McCloskey J. Clasificación de Intervenciones de Enfermería (NIC) $5^{a}$ edición. Barcelona: Elsevier, 2009.

5 Negrete-Salas ME. Distribución geográfica de la población mayor. Demos. Instituto de Investigaciones Sociales. UNAM. 2001, México. pp 18-20.

6 Instituto Nacional de Estadística y Geografía. Censo Nacional de Población y Vivienda 2010. Disponible en: http://bit.ly/igbE59
7 CONAPO. El envejecimiento de la población mundial. Disponible en: http://bit.ly/VhCV5X

8 Genest J, Koiw E, Kuchet O. Hipertensión Arterial. New York: McGraw Hill Book Company. 2005. Pp 345-50.

9 Manuell-Lee G, Gutiérrez-Robledo LM, Gutiérrez-Ávila H. Políticas de salud para los adultos mayores: retos y prioridades. Disponible en: http://bit.ly/XjSAIp

10 Olaiz-Fernández G, Rivera-Dommarco J, Shamah-Levy T, Rojas R, Villalpando-Hernández $\mathrm{S}$, Hernández-Avila M, SepúlvedaAmor J. Encuesta Nacional de Salud y Nutrición 2006. Cuernavaca, México: Instituto Nacional de Salud Pública, 2006.

11 Rivera-Álvarez LN. Capacidad de agencia de autocuidado en personas con hipertensión arterial hospitalizadas en una clínica de Bogotá, Colombia. Rev. salud pública. 2006; 8 (3):235-247.

12 Orem D. Modelo de Orem, Conceptos de enfermería en la práctica. España: Masson - Salvat enfermería, 1993.

13 Cavanagh J. Modelo de Orem. Aplicación práctica. España: Masson Salvat Enfermería. pp. 3-21

14 Coca-Payeras A, Aranda-Lara P, Rendón-Mas J. Manejo del paciente hipertenso en la práctica clínica. Madrid: Ed Médica Panamericana. 2009. Pp. 153-159

15 Ackley B, Ladwing G. Manual de diagnósticos de enfermería.Guía para la planificación de los cuidados. Madrid: Ed ElsevierMosby, 2007. pp. 454, 976, 1128, 1030.

16 De la Cruz-Martínez C. Proceso enfermero en la hipertensión arterial sistémica. Revista Mexicana de Enfermería Cardiológica. 2000; 8 (1-4): 19-24. 


\section{Requisito de desarrollo y en desviación de la salud}

\begin{tabular}{|c|c|c|}
\hline \multicolumn{3}{|c|}{$\begin{array}{l}\text { DIAGNÓSTICO DE ENFERMERIA (NANDA). Mantenimiento i } \\
\text { de conocimientos y/o deterioro cognitivo y/o afrontamien } \\
\text { conocimiento respecto a las prácticas sanitarias básicas. }\end{array}$} \\
\hline RESULTADOS ESPERADOS & $5-N O C$ & ESCALA DE \\
\hline RESULTADOS & - INDICADORES & $\begin{array}{l}\text { MEDICION DEL } \\
\text { INDICADOR }\end{array}$ \\
\hline Conductas de búsqueda de salud & $\begin{array}{l}\text { - Hace preguntas } \\
\text { - Finaliza tareas relacionadas con la salud } \\
\text { - Realiza autodetección } \\
\text { - Obtiene ayuda de un profesional sanitario } \\
\text { - Realiza actividades de la vida diaria compatibles con su } \\
\text { tolerancia } \\
\text { - Describe estrategias para eliminar la conducta insana } \\
\text { - Realiza la conducta sanitaria prescrita } \\
\text { - Utiliza información acreditada de salud } \\
\text { - Describe estrategias para optimizar la salud } \\
\text { - Busca ayuda de familiares cuando es necesario }\end{array}$ & $\begin{array}{l}\text { Nunca demostrado (1), } \\
\text { Siempre demostrado (5) }\end{array}$ \\
\hline $\begin{array}{l}\text { Conducta de fomento } \\
\text { de la salud }\end{array}$ & $\begin{array}{l}\text { - Equilibra actividad y reposo } \\
\text { - Utiliza técnicas efectivas de disminución del estrés } \\
\text { - Sigue una dieta sana } \\
\text { - Obtiene controles regulares } \\
\text { - Utiliza métodos eficaces de control del peso } \\
\text { - Utiliza un programa de ejercicios eficaz }\end{array}$ & $\begin{array}{l}\text { Nunca demostrado (1), } \\
\text { Siempre demostrado (5) }\end{array}$ \\
\hline $\begin{array}{l}\text { Conducta terapéutica: } \\
\text { enfermedad o lesión }\end{array}$ & $\begin{array}{l}\text { - Cumple el régimen terapéutico recomendado } \\
\text { - Cumple el nivel de actividades prescritas } \\
\text { - Cumple el régimen de medicación } \\
\text { - Sigue la dieta prescrita } \\
\text { - Evita conductas que potencian la patología } \\
\text { - Supervisa los efectos terapéuticos } \\
\text { - Supervisa los efectos secundarios del tratamiento } \\
\text { - Supervisa los efectos secundarios de la enfermedad } \\
\text { - Supervisa los cambios en el estado de enfermedad } \\
\text { - Equilibra actividad y reposo } \\
\text { - Obtiene asesoramiento de un profesional cuando es necesario }\end{array}$ & $\begin{array}{l}\text { Nunca demostrado (1), } \\
\text { Siempre demostrado (5) }\end{array}$ \\
\hline $\begin{array}{l}\text { Conocimiento: } \\
\text { conducta sanitaria }\end{array}$ & $\begin{array}{l}\text { - Prácticas nutricionales saludables } \\
\text { - Beneficios de la actividad y el ejercicio } \\
\text { - Efectos de los fármacos de prescripción facultativa } \\
\text { - Servicios de promoción de la salud } \\
\text { - Servicios de protección de la salud }\end{array}$ & $\begin{array}{l}\text { Ningún conocimiento (1), Cono- } \\
\text { cimiento extenso ( } 5 \text { ) }\end{array}$ \\
\hline
\end{tabular}




\section{Enfermería Universitaria}

Conocimiento:

régimen terapéutico

Estado de salud personal

Participación en la decisiones sobre asistencia sanitaria
- Conductas que fomentan la salud

- Estrategias eficaces para hacer frente al estrés

- Prácticas nutricionales saludables

- Medidas eficaces para el control del peso

- Programa de ejercicios eficaz

- Relación entre dieta, ejercicio y peso

- Ejercicio físico

- Nivel de movilidad

- Nivel de energía

- Ejecución de actividades de la vida diaria

- Peso

- Estado nutricional

- Adaptación a condiciones crónicas
Ningún conocimiento (1), Conocimiento extenso (5)
- Reivindica la responsabilidad de tomar decisiones

- Manifiesta autocontrol en la toma de decisiones

- Especifica preferencias de los resultados sanitarios

- Identifica prioridades de los resultados sanitarios

- Declara la intención de actuar según la decisión

- Identifica apoyo disponible para conseguir los resultados deseados

- Busca servicios para cumplir con los resultados deseados
Gravemente comprometido (1), No comprometido (5)

\section{INTERVENCIONES DE ENFERMERIA - NIC}

\section{INTERVENCIONES}

\section{ACTIVIDADES}

- Determinar si hay diferencias entre el punto de vista del paciente y el punto de vista de los cuidadores sanitarios sobre la condición del paciente.

- Ayudar al paciente a aclarar los valores y expectativas que pueden ayudar a tomar decisiones vitales fundamentales.

- Informar al paciente sobre la existencia de puntos de vista alternativos y las soluciones de forma clara y con todo el apoyo.

Apoyo en la toma de decisiones

- Ayudar al paciente a identificar las ventajas y desventajas de cada alternativa.

- Respetar el derecho del paciente a recibir o no información.

- Proporcionar la información solicitada por el paciente.

- Ayudar a que el paciente pueda explicar la decisión a otras personas.

- Servir de enlace entre el paciente y la familia.

- Valorar las razones del paciente para desear cambiar.

- Ayudar al paciente a identificar una meta de cambio específica.

- Ayudar al paciente a identificar conductas objetivo que deban ser cambiadas para conseguir la meta.

- Valorar el nivel de conocimiento y habilidad actual del paciente en relación con el cambio deseado.

- Valorar el ambiente social y físico del paciente por el grado de apoyo de las conductas deseadas.

- Explorar con el paciente las barreras potenciales al cambio de conducta.

- Identificar con el paciente las estrategias más efectivas para el cambio de conducta.

- Explicar al paciente la importancia de la automonitorización en el intento de cambiar la conducta.

- Ayudar al paciente a formular un plan sistemático de cambio de conducta.

- Ayudar al paciente a evaluar el progreso mediante la comparación de conductas previas y actuales.

- Ayudar al paciente a identificar incluso los éxitos más pequeños. 
Educación sanitaria

Enseñanza: proceso de enfermedad
- Identificar factores internos y externos que puedan mejorar o disminuir la motivación en conductas sanitarias.

- Determinar el contexto personal e historial sociocultural de la conducta sanitaria personal o familiar.

- Determinar el conocimiento sanitario actual y las conductas del estilo de vida de los individuos o familias.

- Ayudar a las personas, familia y comunidades para clarificar las creencias y valores sanitarios.

- Formular los objetivos del programa de educación sanitaria.

- Identificar los recursos para llevar a cabo el programa

- Centrarse en beneficios de salud positivos inmediatos o a corto plazo para conductas de estilo de vida positivas, en lugar de beneficios a largo plazo o efectos negativos derivados de incumplimientos.

- Desarrollar materiales educativos escritos en un nivel de lectura adecuado a la audiencia objetivo.

- Mantener una presentación centrada, corta y que comience y termine con el tema principal.

- Implicar al paciente y familia en la planificación e implementación de los planes destinados a la modificación de conductas de estilo de vida o respecto de la salud.

- Planificar un seguimiento a largo plazo para reforzar la adaptación de estilos de vida y conductas saludables.

- Determinar e implementar estrategias para medir los resultados en los pacientes a intervalos regulares durante y después de terminar el programa.

- Evaluar el nivel actual de conocimientos del paciente relacionado con el proceso de enfermedad específico.

- Explicar la fisiopatología de la enfermedad y su relación con la anatomía y fisiología, según cada caso.

- Revisar el conocimiento del paciente sobre su estado.

- Describir los signos y síntomas comunes de la enfermedad, si procede.

- Explorar con el paciente lo que ha hecho para controlar los síntomas

- Describir el proceso de enfermedad, si procede.

- Identificar las etiologías posibles, si procede.

- Proporcionar información al paciente acerca de la enfermedad.

- Evitar las promesas tranquilizadoras vacías.

- Dar seguridad sobre el estado del paciente, si procede.

- Comentar los cambios en el estilo de vida que puedan ser necesarios para evitar futuras complicaciones y/o controlar el proceso de enfermedad.

- Discutir las opciones de tratamiento.

- Describir el fundamento de las recomendaciones del control/ terapia/ tratamiento.

- Animar al paciente a explorar opciones/ conseguir una segunda opinión, si procede o se indica.

- Describir las posibles complicaciones crónicas, si procede.

- Enseñar al paciente medidas para controlar/ minimizar síntomas, si procede.

- Instruir al paciente sobre cuáles son los signos y síntomas de los que debe informarse al cuidador, si procede.

- Reforzar la información otorgada por otros miembros del equipo de cuidados, si procede.
Enseñanza:

procedimiento/ tratamiento
- Reforzar la confianza del paciente en el personal involucrado, si es el caso.

- Determinar las experiencias anteriores del paciente y el nivel de conocimientos relacionados con el tratamiento.

- Explicar el propósito del tratamiento.

- Describir las actividades del tratamiento.

- Explicar el tratamiento.

- Determinar las expectativas del tratamiento por parte del paciente.

- Corregir las expectativas irreales del tratamiento, si procede.

- Discutir tratamientos alternativos, si procede.

- Dar tiempo al paciente para que haga preguntas y discuta sus inquietudes.

- Incluir a la familia/ ser querido, si resulta oportuno. 


\section{Enfermería Universitaria}

2. Requisito universal: Mantenimiento del aporte suficiente de alimento.

DIAGNOSTICO DE ENFERMERIA (NANDA). Desequilibrio nutricional: ingesta superior a las necesidades $\mathrm{r} / \mathrm{c}$ aporte excesivo en relación con las necesidades metabólicas (ingesta de dieta hipercalórica y con gran cantidad de grasa) m/p sobrepeso, sedentarismo y pliegue cutáneo del tríceps > 25 mm en mujeres y >15 mm en varones.

\section{RESULTADOS ESPERADOS - NOC}

\section{RESULTADOS}

\section{INDICADORES}

Ingestión calórica

Estado nutricional: Ingestión de nutrientes

Conducta de adhesión: dieta saludable

Conocimiento: Dieta

Conocimiento: manejo del peso

Conducta de pérdida de peso

Motivación

\section{Ingestión proteica}

Ingestión de grasas

Ingestión de sodio

\section{ESCALA DE}

\section{MEDICION}

\section{DEL INDICADOR}

Inadecuado (1),

Completamente adecuado (5)
Establece objetivos dietéticos alcanzables Selecciona raciones compatibles con las pautas nutricionales recomendadas

Come las raciones de fruta recomendadas cada día Come las raciones de verdura recomendadas cada día Minimiza la ingesta de alimentos con alto contenido calórico y poco valor nutricional

\section{Dieta recomendada}

Fundamentos de la dieta recomendada

Ventajas de seguir la dieta recomendada

Objetivos para la dieta

Relaciones entre dieta, ejercicio y peso corporal

Comidas permitidas en la dieta

Líquidos permitidos en la dieta

Comidas que deben evitarse

Líquidos que deben evitarse

Peso personal óptimo

Índice de Masa Corporal (IMC) óptimo

Estrategias para conseguir el peso óptimo

Estrategias para mantener el peso óptimo

Ningún conocimiento (1),

Riesgos de salud relacionados con el sobrepeso

Beneficios de la actividad física y el ejercicio

Obtiene información de un profesional sanitario

sobre estrategias para perder peso

Se compromete con un plan para comer de forma saludable

Controla la ración de alimentos

Establece una rutina de ejercicios

El gasto calórico supera la ingesta calórica

Identifica estados emocionales que afectan

la ingesta de alimentos y líquidos

Utiliza estrategias de modificación de la conducta

Evita la ingesta de alimentos y líquidos muy calóricos

Bebe ocho vasos de agua al día

Obtiene el apoyo necesario

Autoinicia conductas dirigidas hacia los objetivos

Expresa la creencia en la capacidad para realizar la acción

Expresa que la ejecución conducirá al resultado deseado

Finaliza las tareas

Nunca demostrado (1),

Acepta las responsabilidades de las acciones

Expresa intención de actuar
Ningún conocimiento (1),

Conocimiento extenso (5)
Nunca demostrado (1),

Siempre demostrado (5) 


\section{INTERVENCIONES DE ENFERMERIA - NIC INTERVENCIONES ACTIVIDADES}

Asesoramiento nutricional

- Determinar la ingesta y los hábitos alimentarios del paciente.

- Ayudar al paciente a registrar lo que suele comer en un período de 24 horas.

- Facilitar la identificación de las conductas alimentarias que se desean cambiar.

- Establecer metas realistas a corto y largo plazo para el cambio del estado nutricional.

- Proporcionar información acerca de la necesidad de modificación de la dieta por razones de salud: pérdida de peso, restricción de sodio, reducción de colesterol, restricción de líquidos, etc.

- Ayudar al paciente a considerar los factores de enfermedades, cultura y economía en la planificación de las formas de cumplir con las necesidades nutricionales.

- Determinar el conocimiento por parte del paciente de los cuatro grupos alimenticios básicos, así como la percepción de la necesidad de modificación de la dieta.

- Discutir las necesidades nutricionales y la percepción del paciente de la dieta prescrita/ recomendada.

- Revisar con el paciente la medición de ingesta y eliminación de líquidos, lecturas de presión sanguínea o ganancias y pérdidas de peso, si procede.

- Discutir el significado de la comida para el paciente

- Determinar las actitudes y creencias de los seres queridos acerca de la comida, el comer y el cambio nutricional del paciente.

Ayuda para disminuir el peso

- Valorar el progreso de las metas de modificación dietética a intervalos regulares.

- Valorar los esfuerzos realizados para conseguir los objetivos.

- Disponer una visita/consulta a otros miembros del equipo de cuidados, si procede.

- Determinar el deseo y la motivación del paciente para reducir el peso o la grasa corporal.

- Determinar con el paciente la cantidad de pérdida de peso deseada.

- Pesar al paciente regularmente

- Registrar el progreso para llegar a la meta final y colocarla en un sitio estratégico.

- Fomentar el uso de sistemas de recompensa internos cuando se alcanzan las metas.

- Establecer un plan realista con el paciente que incluya la disminución en la ingesta de alimentos y el aumento del gasto de energía.

- Determinar los esquemas de alimentación actuales haciendo que el paciente lleve un diario de lo que come, cuándo y dónde.

- Fomentar la sustitución de hábitos indeseables por hábitos favorables.

- Ayudar al paciente a ajustar la dieta al estilo de vida y nivel de actividad.

- Planificar un programa de ejercicios, teniendo en cuenta las limitaciones del paciente.

- Desarrollar un plan de comidas diarias con una dieta bien equilibrada, disminución de calorías y de grasa, si procede.

- Fomentar el uso de sustitutos de azúcar si procede. 


\section{Enfermería Universitaria}

Enseñanza: Dieta prescrita

Enseñanza: Actividad /ejercicio prescrito.
- Evaluar el nivel actual de conocimientos del paciente acerca de la dieta prescrita.

- Conocer los sentimientos/ actitud del paciente/ ser querido acerca de la dieta. prescrita y del grado de cumplimiento dietético esperado.

- Explicar al paciente el propósito de la dieta.

- Instruir al paciente sobre los alimentos permitidos y los alimentos que deben evitarse

- Ayudar al paciente a acomodar sus preferencias de comidas en la dieta prescrita.

- Proporcionar un plan escrito de comidas, si procede.

- Remitir al paciente a un dietista/ experto en nutrición, si es preciso.

- Reforzar la información proporcionada por otros miembros del equipo de cuidados, según corresponda.

- Incluir a la familia/ ser querido, según sea el caso.

- Evaluar el nivel actual de ejercicio del paciente y conocimiento de la actividad/ ejercicio prescrito.

- Informar al paciente del propósito y los beneficios de la actividad/ ejercicio prescrito.

- Enseñar al paciente a realizar la actividad/ ejercicio prescrito.

- Enseñar al paciente a llevar un diario de ejercicios, si resulta posible.

- Informar al paciente acerca de las actividades apropiadas en función del estado físico.

- Instruir al paciente acerca de los ejercicios de estiramiento correctos antes y después de la actividad/ ejercicio y el fundamento de tal acción, si procede.

- Enseñar al paciente a realizar un precalentamiento y enfriamiento antes y después de la actividad/ ejercicio y la importancia de tal acción, si procede.

- Enseñar al paciente una buena postura y mecánica corporal durante el ejercicio.

- Observar al paciente mientras realiza la actividad/ejercicio.

- Ayudar al paciente a incorporar la actividad/ejercicio en la rutina diaria/estilo de vida.

- Remitir al paciente al fisioterapeuta/ terapeuta ocupacional, si procede.

- Reforzar la información proporcionada por otros miembros del equipo de cuidados.

- Incluir a la familia / ser querido, si resulta apropiado.

- Remitir al paciente a un centro de rehabilitación, si se precisa.

- Valorar las ideas del individuo sobre el efecto del ejercicio físico en la salud.

- Explorar experiencias deportivas anteriores.

- Determinar la motivación del individuo para empezar/ continuar con el programa de ejercicios.

- Explorar las barreras para el ejercicio.

- Fomentar la manifestación oral de sentimientos acerca de los ejercicios o la necesidad de los mismos.

- Animar al individuo a empezar o continuar con el ejercicio.

- Ayudar al individuo a desarrollar un programa de ejercicios adecuado a sus necesidades.

- Ayudar al individuo a establecer las metas a corto y largo plazo del programa de ejercicios.

- Ayudar al individuo a integrar el programa de ejercicios a su rutina semanal.

- Incluir a la familia /cuidadores del paciente en la planificación y mantenimiento del programa de ejercicios.

Fomento del ejercicio

- Informar al individuo acerca de los beneficios para la salud y los efectos psicológicos del ejercicio.

- Instruir al individuo acerca del tipo de ejercicio adecuado para su nivel de salud, en colaboración con el médico y/o fisioterapeuta.

- Instruir al individuo acerca de la frecuencia, duración e intensidad deseadas de los ejercicios del programa.

- Controlar el cumplimiento del individuo del programa de ejercicios.

- Instruir al individuo acerca de las condiciones que justifiquen el cese o alteración del programa de ejercicios.

- Instruir al individuo en los ejercicios de precalentamiento y relajación adecuados.

- Preparar al individuo en técnicas para evitar lesiones durante el ejercicio.

- Reforzar el programa para potenciar la motivación del paciente (ej. Estimación del aumento de la resistencia, pesajes semanales, etc.)

- Proporcionar una respuesta positiva a los esfuerzos del individuo. 


\section{Requisito: Mantenimiento de balance entre actividad y reposo}

DIAGNOSTICO DE ENFERMERIA (NANDA). Sedentarismo r/c falta de interés y/o falta de motivación
y/o conocimientos deficientes sobre los beneficios del ejercicio físico para la salud m/p
falta de condición física, verbaliza preferencia por actividades con poca movilidad física
y rutina diaria con falta de ejercicio físico
RESULTADOS ESPERADOS - NOC
EESULTADOS INDICADORES
MEDICION DEL
INDICADOR

Conducta de cumplimiento

Estado de salud personal

Forma física

Nivel de fatiga

Resistencia
Acepta el diagnóstico del profesional sanitario

Busca información acreditada sobre el diagnóstico

Busca información acreditada sobre el tratamiento

Refiere los cambios de síntomas a un profesional sanitario

Realiza las actividades de la vida diaria según prescripción

Busca ayuda externa para ejecutar la conducta sanitaria

Ejercicio físico

Nivel de movilidad

Nivel de energía

Ejecución de actividades de la vida diaria

Peso

Estado nutricional

Adaptación a condiciones crónicas

Flexibilidad articular

Participación en actividades físicas

Ejercicio habitual

Función cardiovascular

Función respiratoria

Gravemente comprometido

Forma física aeróbica

Índice de masa corporal

Presión arterial

Frecuencia cardiaca durante el ejercicio

Frecuencia cardiaca en reposo

Agotamiento

Estado de ánimo deprimido

Descenso de la motivación

Cefalea

Mialgia

Artralgia

Malestar después del ejercicio

Nivel de estrés

Grave (1),

Ninguno (5)

Actividades de la vida diaria

Actividades instrumentales de la vida diaria

Calidad del descanso

Calidad del sueño

Equilibrio actividad/ reposo

Realización de la rutina habitual

Actividad

Resistencia muscular

Gravemente comprometido

Recuperación de la energía con el descanso
Nunca demostrado (1),

Siempre demostrado (5)

Gravemente comprometido (1), No comprometido (5)
(1), No comprometido (5) 


\section{Enfermería Universitaria}

Tolerancia de la actividad

Conducta de adhesión

Conocimiento de fomento de la salud

Conducta de fomento de la salud

Motivación
Frecuencia cardíaca en respuesta a la actividad

Frecuencia respiratoria en respuesta a la actividad

Esfuerzo respiratorio en respuesta a la actividad

Paso al caminar

Distancia de caminata

Gravemente comprometido

Tolerancia al subir escaleras

Resistencia de la parte superior del cuerpo

Resistencia de la parte inferior del cuerpo

Facilidad para realizar las actividades de la vida diaria (AVD)

Habilidad para hablar durante el ejercicio

Realiza preguntas

Contrapesa los riesgos y los beneficios de la conducta sanitaria

Refiere el uso de estrategias para optimizar al máximo la salud

Utiliza los servicios de atención sanitaria de forma congruente con las necesidades

Realiza actividades de la vida diaria compatibles

con su energía y tolerancia

Conductas que fomentan la salud

Estrategias eficaces para hacer frente al estrés

Prácticas nutricionales saludables

Medidas eficaces para el control del peso

Programa de ejercicios eficaz

Relación entre dieta, ejercicio y peso

Equilibra actividad y reposo

Utiliza técnicas efectivas de disminución del estrés

Sigue una dieta sana

Obtiene controles regulares

Utiliza métodos eficaces de control del peso

Utiliza un programa de ejercicios eficaz

Obtiene el apoyo necesario

Autoinicia conductas dirigidas hacia los objetivos

Expresa la creencia en la capacidad para realizar la acción

Expresa que la ejecución conducirá al resultado deseado

Finaliza las tareas

Nunca demostrado (1),

Siempre demostrado (5)
Nunca demostrado (1), Siempre demostrado (5)

Ningún conocimiento (1),

Conocimiento extenso (5)

Nunca demostrado (1), Siempre demostrado (5)

Acepta las responsabilidades de las acciones

Expresa intención de actuar

\section{INTERVENCIONES DE ENFERMERIA - NIC}

\section{INTERVENCIONES}

Enseñanza:

Actividad/ejercicio prescrito.

\section{ACTIVIDADES}

- Evaluar el nivel actual de ejercicio del paciente y conocimiento de la actividad/ ejercicio prescrito.

- Informar al paciente del propósito y los beneficios de la actividad/ ejercicio prescrito.

- Enseñar al paciente a realizar la actividad/ ejercicio prescrito.

- Enseñar al paciente a llevar un diario de ejercicios, si resulta posible.

- Informar al paciente acerca de las actividades apropiadas en función del estado físico.

- Instruir al paciente acerca de los ejercicios de estiramiento correctos antes y después de la actividad/ ejercicio y el fundamento de tal acción, si procede.

- Enseñar al paciente a realizar un precalentamiento y enfriamiento antes y después de la actividad/ ejercicio y la importancia de tal acción, si procede.

- Enseñar al paciente una buena postura y mecánica corporal durante el ejercicio.

- Observar al paciente mientras realiza la actividad/ejercicio.

- Ayudar al paciente a incorporar la actividad/ejercicio en la rutina diaria/estilo de vida.

- Remitir al paciente al fisioterapeuta/ terapeuta ocupacional, si procede.

- Reforzar la información proporcionada por otros miembros del equipo de cuidados.

- Incluir a la familia / ser querido, si resulta apropiado.

- Remitir al paciente a un centro de rehabilitación, si se precisa. 


\section{Fomento del ejercicio}

\section{Manejo de la energía}

Modificación de la conducta
- Valorar las ideas del individuo sobre el efecto del ejercicio físico en la salud.

- Explorar experiencias deportivas anteriores.

- Determinar la motivación del individuo para empezar/ continuar con el programa de ejercicios.

- Explorar las barreras para el ejercicio.

- Fomentar la manifestación oral de sentimientos acerca de los ejercicios o la necesidad de los mismos.

- Animar al individuo a empezar o continuar con el ejercicio.

- Ayudar al individuo a desarrollar un programa de ejercicios adecuado a sus necesidades.

- Ayudar al individuo a establecer las metas a corto y largo plazo del programa de ejercicios.

- Ayudar al individuo a integrar el programa de ejercicios a su rutina semanal.

- Incluir a la familia /cuidadores del paciente en la planificación y mantenimiento del programa de ejercicios.

- Informar al individuo acerca de los beneficios para la salud y los efectos psicológicos del ejercicio.

- Instruir al individuo acerca del tipo de ejercicio adecuado para su nivel de salud, en colaboración con el médico y/o fisioterapeuta.

- Instruir al individuo acerca de la frecuencia, duración e intensidad deseadas de los ejercicios del programa.

- Controlar el cumplimiento del individuo del programa de ejercicios.

- Instruir al individuo acerca de las condiciones que justifiquen el cese o alteración del programa de ejercicios.

- Instruir al individuo en los ejercicios de precalentamiento y relajación adecuados.

- Preparar al individuo en técnicas para evitar lesiones durante el ejercicio.

- Reforzar el programa para potenciar la motivación del paciente (ej. Estimación del aumento de la resistencia, pesajes semanales, etc.)

- Proporcionar una respuesta positiva a los esfuerzos del individuo.

- Determinar los déficits en el estado fisiológico del paciente que producen fatiga según el contexto de la edad.

- Animar a la verbalización de los sentimientos sobre las limitaciones.

- Determinar la percepción de la causa de fatiga por parte del paciente/ ser querido.

- Determinar qué actividad y en qué medida es necesaria para aumentar la resistencia.

- Controlar la ingesta nutricional para asegurar recursos energéticos adecuados.

- Vigilar la respuesta cardiorrespiratoria a la actividad (taquicardia, otras disritmias, disnea, diaforesis, palidez y frecuencia respiratoria).

- Animar a realizar ejercicios aeróbicos, según la tolerancia.

- Observar/ registrar el esquema y número de horas de sueño del paciente.

- Observar la localización y naturaleza de la molestia o dolor durante el movimiento/ actividad.

- Ayudar al paciente/ ser querido a establecer metas realistas de actividades.

- Ayudar al paciente a identificar las preferencias de actividades.

- Ayudar al paciente a elegir actividades que reconstruyan la resistencia de forma gradual.

- Facilitar la alternancia de períodos de reposo y actividad.

- Favorecer la actividad física (deambulación o realización de actividades de la vida diaria), coherente con los recursos energéticos del paciente.

- Instruir al paciente y/o los seres queridos sobre el estrés e intervenciones de enfrentamiento para disminuir la fatiga.

- Determinar la motivación al cambio del paciente.

- Ayudar al paciente a identificar su fortaleza y reforzarla.

- Fomentar la sustitución de hábitos indeseables por hábitos deseables.

- Evitar mostrar rechazo o restar importancia a los esfuerzos del paciente por cambiar su conducta.

- Ofrecer un refuerzo positivo a las decisiones del paciente que han sido tomadas independientemente.

- Establecer objetivos de conducta de forma escrita.

- Facilitar la implicación familiar en el proceso de modificación de conducta. 


\section{Enfermería Universitaria}

\section{Requisito en desviación de la salud}

\section{DIAGNOSTICO DE ENFERMERIA (NANDA). Déficit de conocimientos con respecto a HTA}

y signos y síntomas de alarma $r / c$ la falta de exposición y/o deterioro de memoria

y/o mala interpretación de la información y/o limitación cognitiva y/o falta de interés en el aprendizaje

y/o poco familiaridad con los recursos para obtener información m/p verbalización del problema y/o se-

\section{guimiento inexacto de las instrucciones (tratamiento).}

\section{RESULTADOS ESPERADOS - NOC}

\section{RESULTADOS INDICADORES}

\section{ESCALA DE}

MEDICION DEL

INDICADOR
Conocimiento: control de la hipertensión
Rango normal de presión arterial sistólica

Rango normal de presión arterial diastólica

Métodos para medir la presión arterial

Complicaciones potenciales de la hipertensión

Importancia del tratamiento a largo plazo

Signos y síntomas de exacerbación de la hipertensión

Uso correcto de la medicación prescrita

Efectos terapéuticos de la medicación

Efectos secundarios de la medicación

Efectos adversos de la medicación

Importancia del seguimiento de la medicación

Importancia de informar al profesional sanitario toda la medicación

actual

Importancia de mantener visitas de seguimiento

Beneficios del autocontrol continuo

Programa recomendado para controlar la presión arterial

Beneficios de la pérdida de peso

Beneficios de las modificaciones del estilo de vida

Estrategias para controlar el estrés

Modificaciones de dieta recomendadas

Estrategias para cambiar los hábitos dietéticos

Estrategias para limitar la ingesta de sodio

Estrategias para potenciar el seguimiento de la dieta

Efectos del consumo de alcohol

Importancia de la abstinencia de tabaco

Beneficios de la actividad y el ejercicio

Cuando obtener ayuda de un profesional sanitario

Beneficios del manejo de la enfermedad
Ningún conocimiento (1), Conocimiento extenso (5)
Importancia de informar al profesional sanitario toda la medicación que se está consumiendo

Declaración del nombre correcto de la medicación

Efectos terapéuticos de la medicación

Conocimiento: medi- Efectos indeseables de la medicación cación
Efectos adversos de la medicación

Uso de sistemas de ayuda para recordar

Posibles reacciones adversas cuando se consumen múltiples fármacos

Administración correcta de la medicación

Técnicas de automonitorización
Ningún conocimiento (1), Conocimiento extenso (5) 
Justificación del régimen terapéutico

Responsabilidad de los propios cuidados para el tratamiento actual

Técnicas de automonitorización

Conocimientos:

Régimen terapéutico

Efectos esperados del tratamiento

Dieta prescrita

Medicación prescrita

Actividad prescrita

Ejercicio prescrito

Beneficios del tratamiento de la enfermedad
Ningún conocimiento (1), Conocimiento extenso (5)
Comunicación clara según edad

Comunicación adecuada según edad

Comprende el significado de situaciones

Atiende

Cognición

Se concentra

Está orientado

Memoria inmediata

Memoria reciente

Memoria remota

Compara las alternativas al tomar decisiones
Gravemente comprometido (1), No comprometido (5)
Memoria

Recuerda información inmediata de forma precisa

Recuerda información reciente de forma precisa

Recuerda información remota de forma precisa
Gravemente comprometido (1), No comprometido (5)
Obtiene el apoyo necesario

Autoinicia conductas dirigidas hacia los objetivos

Expresa la creencia en la capacidad para realizar la acción

Motivación
Expresa que la ejecución conducirá al resultado deseado

Finaliza las tareas

Acepta las responsabilidades de las acciones

Expresa intención de actuar
Nunca demostrado (1), Siempre demostrado (5) 


\section{Enfermería Universitaria}

\section{INTERVENCIONES DE ENFERMERIA - NIC}

\section{INTERVENCIONES ACTIVIDADES}

- Establecer la credibilidad del educador.

- Determinar las necesidades de enseñanza del paciente.

- Valorar el nivel actual de conocimientos y comprensión de contenidos del paciente.

- Valorar el nivel educativo del paciente.

- Valorar las capacidades/ incapacidades cognoscitivas, psicomotoras y afectivas.

- Determinar la capacidad del paciente para asimilar información específica.

- Determinar la motivación del paciente para asimilar información específica.

- Potenciar las disponibilidad del paciente para aprender, si procede.

Enseñanza: individual

- Identificar los objetivos de enseñanza necesarios para conseguir las metas.

- Determinar la secuencia de presentación de la información.

- Seleccionar los métodos/ estrategias de enseñanza para el paciente.

- Seleccionar los materiales educativos adecuados.

- Corregir las malas interpretaciones de la información, si procede.

- Dar tiempo al paciente para que haga preguntas y discuta sus inquietudes.

- Seleccionar nuevos métodos/ estrategias de enseñanza, si los anteriores hubieran sido ineficaces.

- Remitir al paciente a otros especialistas/ centros para conseguir los objetivos de enseñanza, si es preciso.

- Registrar el contenido presentado, los materiales escritos proporcionados y la comprensión del paciente de la información o las conductas del paciente que indiquen aprendizaje en el registro médico

- Identificar factores internos y externos que puedan mejorar o disminuir la motivación en conductas sanitarias.

- Determinar el contexto personal e historial sociocultural de la conducta sanitaria personal o familiar.

- Determinar el conocimiento sanitario actual y las conductas del estilo de vida de los individuos o familias.

- Ayudar a las personas, familia y comunidades para clarificar las creencias y valores sanitarios.

- Formular los objetivos del programa de educación sanitaria.

- Identificar los recursos para llevar a cabo el programa

Educación sanitaria

- Centrarse en beneficios de salud positivos inmediatos o a corto plazo para conductas de estilo de vida positivas, en lugar de beneficios a largo plazo o efectos negativos derivados de incumplimientos.

- Desarrollar materiales educativos escritos en un nivel de lectura adecuado a la audiencia objetivo.

- Mantener una presentación centrada, corta y que comience y termine con el tema principal.

- Implicar al paciente y familia en la planificación e implementación de los planes destinados a la modificación de conductas de estilo de vida o respecto de la salud.

- Planificar un seguimiento a largo plazo para reforzar la adaptación de estilos de vida y conductas saludables.

- Determinar e implementar estrategias para medir los resultados en los pacientes a intervalos regulares durante y después de terminar el programa.

- Evaluar el nivel actual de conocimientos del paciente relacionado con el proceso de enfermedad específico.

- Explicar la fisiopatología de la enfermedad y su relación con la anatomía y fisiología, según cada caso.

- Revisar el conocimiento del paciente sobre su estado.

- Describir los signos y síntomas comunes de la enfermedad, si procede.

- Explorar con el paciente lo que ha hecho para controlar los síntomas

- Describir el proceso de enfermedad, si procede.

- Identificar las etiologías posibles, si procede.

- Proporcionar información al paciente acerca de la enfermedad.

- Evitar las promesas tranquilizadoras vacías.

- Dar seguridad sobre el estado del paciente, si procede.

- Comentar los cambios en el estilo de vida que puedan ser necesarios para evitar futuras complicaciones y/o controlar el proceso de enfermedad.

- Discutir las opciones de tratamiento.

- Describir el fundamento de las recomendaciones del control/ terapia/ tratamiento.

- Animar al paciente a explorar opciones/ conseguir una segunda opinión, si procede o se indica.

- Describir las posibles complicaciones crónicas, si procede.

- Enseñar al paciente medidas para controlar/ minimizar síntomas, si procede.

- Instruir al paciente sobre cuáles son los signos y síntomas de los que debe informarse al cuidador, si procede.

- Reforzar la información otorgada por otros miembros del equipo de cuidados, si procede. 
Enseñanza: procedimiento/ tratamiento
- Reforzar la confianza del paciente en el personal involucrado, si es el caso.

- Determinar las experiencias anteriores del paciente y el nivel de conocimientos relacionados con el tratamiento.

- Explicar el propósito del tratamiento.

- Describir las actividades del tratamiento.

- Explicar el tratamiento.

- Determinar las expectativas del tratamiento por parte del paciente.

- Corregir las expectativas irreales del tratamiento, si procede.

- Discutir tratamientos alternativos, si procede.

- Dar tiempo al paciente para que haga preguntas y discuta sus inquietudes.

- Incluir a la familia/ ser querido, si resulta oportuno.

- Animar al paciente a que determine sus virtudes y habilidades.

- Ayudar al paciente a identificar las prácticas sobre la salud que desea cambiar.

- Determinar con el paciente los objetivos de los cuidados.

- Al ayudar al paciente a identificar las metas, evitar centrarse en el diagnóstico o proceso de enfermedad únicamente.

Acuerdo con el paciente - Ayudar al paciente a establecer objetivos realistas, que puedan conseguirse.

- Ayudar al paciente a desarrollar un plan para cumplir con los objetivos.

- Ayudar al paciente a establecer las necesidades de tiempo y de frecuencia para la realización de las conductas/ acciones.

- Ayudar al paciente a identificar hasta los más pequeños éxitos.

- Explorar con el paciente las razones del éxito o la falta de éste.

- Enseñar al paciente a reconocer las características distintivas del (de los) medicamento(s), si procede.

- Informar al paciente tanto el nombre genérico como el comercial de cada medicamento.

- Informar al paciente acerca del propósito y acción de cada medicamento.

- Instruir al paciente sobre la dosis, vía y duración de los efectos de cada medicamento.

- Revisar el conocimiento que el paciente tiene de las medicaciones.

- Evaluar la capacidad del paciente para administrarse los medicamentos el mismo.

- Informar al paciente sobre lo que tiene que hacer si pasa por alto una dosis.

- Informar al paciente sobre las consecuencias de no tomar o suspender bruscamente la medicación.

- Instruir al paciente sobre los posibles efectos adversos de cada medicamento.

- Enseñar al paciente a aliviar o prevenir ciertos efectos secundarios, si es el caso.

- Instruir al paciente sobre los signos y síntomas de sobredosis o subdosis.

- Ayudar al paciente a desarrollar un horario de medicación escrito.

- Advertir al paciente de los riesgos asociados a la administración de medicamentos caducados.

- Advertir al paciente contra la administración de medicamentos prescritos a otras personas.

- Incluir a la familia/ ser querido, si procede.
Ayuda para dejar de fumar
- Registrar el estado actual y el historial de fumar.

- Determinar la facilidad del paciente para aprender sobre dejar de fumar.

- Aconsejar al fumador de forma clara y consistente dejar de fumar.

- Ayudar al paciente a identificar las razones para dejar de fumar y las barreras.

- Enseñar al paciente los síntomas físicos de la abstinencia de la nicotina.

- Asegurar al paciente que los síntomas físicos de abstinencia son temporales.

- Informar al paciente sobre productos sustitutivos de la nicotina para ayudar a reducir los síntomas físicos de la abstinencia

- Ayudar al paciente a desarrollar métodos prácticos para resistir las ansias de fumar

- Animar al paciente a mantener un estilo de vida sin tabaco 